\title{
Survey of Wildlife Rehabilitators on Infection Control and Personal Protective Behaviors
}

\author{
Emi K. Saito and Allison A. Shreve \\ National Wildlife Health Center, US Geological Survey, Madison, Wisconsin
}

\begin{abstract}
Wildlife veterinarians and rehabilitators treat a number of wildlife species that can carry infectious and zoonotic diseases. These can rapidly spread within a facility and to the caregivers when adequate measures are not taken. Financial constraints and reduced access to laboratories often limit identification of disease etiology of many cases admitted into wildlife rehabilitation centers. A survey to investigate willdife rehabilitator illness during the 2002 West Nile virus season indicated that many rehabilitators do not follow adequate protective measures in their facilities and may not seek medical care when disease symptoms arise or even linger. The recommended precautions and proper infection control measures for those handling and housing wildlife cases should be discussed between rehabilitators and their attending veterinarian(s).
\end{abstract}

Key Words: zoonoses; disease prevention; personal protection; disinfection; survey; West Nile virus; mosquito protection

\section{INTRODUCTION}

During the 2002 West Nile virus (WNV) season an influx of raptor cases was reported to the US Geological Survey National Wildlife Health Center (NWHC), Madison, Wisconsin, by wildlife officials and wildlife rehabilitation facilities in several states, particularly those reporting high WNV activity. The high profile of WNV in these regions and the clinical presentations of sick raptors led many rehabilitators to assume the increased admissions of raptors were due to WNV infection. Shortly afterward, the NWHC received reports of rehabilitator illness thought to be due to WNV. Some of these illnesses were diagnosed by physicians as WNV infection. Many rehabilitators believed they had been directly infected by treating WNV-infected birds and not by mosquito exposure. An investigation was initiated by NWHC in November 2002 to assess the incidence of WNV-like illness in rehabilitators in WNV-affected areas. In addition, these cases were examined to determine the

Dr. Saito is currently affiliated with the US Department of Agriculture, Animal and Plant Health Inspection Service, Veterinary Services, Center for Epidemiology and Animal Health, Natlional Center for Animal Health Surveillance, Ames, IA

Dr. Shreve is currently affiliated with the Wildlife Care Center, Fort Lauderdale, FL likelihood infection had been acquired by direct contact with sick birds.

\section{MATERIALS AND METHODS}

The NWHC received reports of increased raptor admissions during the 2002 WNV season from 14 states: Illinois, Indiana, Iowa, Kentucky, Louisiana, Maryland, Michigan, Minnesota, Missouri, New Jersey, Ohio, Pennsylvania, Virginia, and Wisconsin. Wildlife rehabilitators from these states were randomly selected from the 2002 National Wildlife Rehabilitators Association (NWRA) Membership Directory. A telephone survey was conducted in November 2002 to determine the number of rehabilitators diagnosed with WNV and to assess the possibility of direct birdto-human transmission of the virus by examining factors such as infection control procedures and exposure to mosquitos. A person with illness was considered to be a WNV case only if diagnosed by a physician.

Participants provided data for the 2002 WNV season with regard to: approximate number of admitted raptor cases (overall), approximate number of WNV raptor cases (suspect or confirmed), personal protection practices employed while treating and handling wildlife, disinfection practices utilized at the facilities, mosquito protection practices, and whether or not the rehabilitator had experienced illness. Logistic regression analyses were performed using SAS v8 (SAS Institute, Cary, NC) to evaluate the relationship between the number of mosquito bites received and mosquito protection practiced, and between illness and: 1) the number of suspected or confirmed WNV cases treated; 2) personal protection measures followed while handling sick birds; 3 ) the number of mosquito bites received; and 4) facility disinfection practices.

\section{RESULTS}

A total of 109 wildlife rehabilitators from the 14 aforementioned states were randomly selected from the NWRA directory. Eighty of the selected rehabilita- 
tors were reached, and 42 agreed to participate in the survey. Thirty-four $(81.0 \%)$ of the participants were female. Other demographic, raptor rehabilitation, and rehabilitator health information is shown in Table 1. Nonspecific symptoms that could have been due to WNV infection were reported in nine respondents, but only one (11.1\%) sought medical care, after which the diagnosis was unknown. One participant reported that the symptoms could have been stress-related. The most commonly reported symptoms were headache and myalgia. Other symptoms reported included: weakness, tremors, fever, sweats and chills, enlarged lymph nodes, dizziness, nausea, and diarrhea. All but two reported at least three symptoms; the two participants reported only myalgia. Three participants reported fever, headaches and myalgia; one of these was the participant who sought medical care. At the time of the survey (at least two months post-onset), two participants reported that they still had some residual symptoms and had not yet sought medical care.

Personal protection practices of the respondents and disinfection practices at the rehabilitation facilities varied greatly (Table 2 ). Common personal protective measures against injury and exposure to contaminated materials included wearing gloves (leather and/or latex) and hand washing (frequency not always clarified). There were 23 participants who reported using both latex and leather gloves, although it was not determined if and when they wore both

Table 1. Raptor rehabilitator information $(n=42)$ in November 2002. Data on rehabilitation cases reflect only those admitted during 2002 WNV season.

\begin{tabular}{|c|c|c|}
\hline Rehabilitator Information & Median & Range \\
\hline Age & 46.5 & $23-74$ \\
\hline Female $(n=34)$ & 48.5 & 24-65 \\
\hline Male $(n=8)$ & 32 & $23-74$ \\
\hline \# Years rehabilitation experience & 10 & Feb-50 \\
\hline \# Raptor cases during 2002 WNV season & 32.5 & $2-760$ \\
\hline \# WNV Cases (suspect or confirmed) & 10.75 & $1-100$ \\
\hline Average time spent with raptors (hrs/day) & 1.5 & $0.2-7$ \\
\hline Average time spent outdoors (hrs/day) & 4.8 & $0.3-15$ \\
\hline & \multicolumn{2}{|c|}{ Frequency $(\%)$} \\
\hline \# Admitting Suspect/Confirmed WNV Raptors & \multicolumn{2}{|c|}{81} \\
\hline \# Developing illness & \multicolumn{2}{|c|}{21.4} \\
\hline Headaches & \multicolumn{2}{|c|}{16.8} \\
\hline Myalgia & \multicolumn{2}{|c|}{19} \\
\hline Weakness & \multicolumn{2}{|c|}{7.1} \\
\hline Tremors & \multicolumn{2}{|c|}{4.8} \\
\hline Other signs & \multicolumn{2}{|c|}{19} \\
\hline
\end{tabular}


together. Three respondents reported using neither type of gloves: one who only practiced handwashing and two who reported practicing no protective behaviors against infection or injury. All 20 respondents who reported injury (scratches, bites) reported always wearing leather gloves, with the exception of one individual who used only latex gloves, while handling raptors. Masks, face shields and/or goggles were used to protect against aerosol or body fluid exposure on an "as needed" basis, if at all. The three respondents that followed no personal protection practices also reported no injuries.

Disinfection procedures at facilities varied, although most (81.0\%) followed some disinfection practices. One of the cleaning products used by four respondents is sold in a cleaning formulation, as well as in a disinfectant formulation; it was assumed for the purposes of this survey that the disinfectant for- mulation was used by these participants. The frequency of cage disinfection varied: 15 respondents $(35.7 \%)$ reported daily cage disinfection, 14 (33.3\%) reported cage disinfection only between cases, and five (11.9\%) reported disinfecting cages only on a periodic basis. One participant did not know what disinfection practices were followed at their facility. Three participants indicated cleaning was done daily, but were unable to specify the cleaning agent used. Use of solutions not intended for disinfecting or cleaning purposes was reported by two rehabilitators who reported cleaning cages daily and two who cleaned cages only between cases.

Mosquito bites were reported by 39 (92.9\%) respondents. Among 34 respondents, the median number of mosquito bites received was 11 (range: 0-250). Half of the respondents reported using no protection against mosquito exposure, despite some

Table 2. Personal protective and infection control measures followed by participants $(n=42)$. Frequencies presented are based on sometimes or always follow measures (vs. never).

$\begin{array}{lc}\text { Animal-handling Protective Measures } & \text { Frequency (\%) } \\ \text { Leather gloves } & 88.1 \\ \text { Latex gloves } & 66.7 \\ \text { Face mask } & 26.2 \\ \text { Goggles } & 7.1 \\ \text { Hand washing } & 45.2 \\ \text { \# Reporting any wounds associated with handling } & 47.6 \\ \text { Disinfection of Materials } & \text { Frequency (\%) } \\ \text { Method of cage-cleaning } & \\ \text { Disinfecting solution, including bleach } & 78.6 \\ \text { Non-cleaning solution } & 9.5 \\ \text { Unsure/None } & 11.9 \\ \text { Cages, daily } & 35.7 \\ \text { Cages, between cases only } & 33.3 \\ \text { Protective Measures against Mosquitoes } & \text { Frequency (\%) } \\ \text { Any mosquito protection } & 50 \\ \text { Method of protection } & \\ \quad \text { DEET-based product } & 38.1 \\ \text { Clothing } & 16.7 \\ \text { Other } & 4.8\end{array}$


reporting an uncountable number of mosquito bites that season. Of the 21 respondents who practiced some form of protection against mosquito exposure, $17(81 \%)$ reported always following these measures when outdoors. Only one respondent reported both using mosquito repellent and wearing protective clothing while outdoors. The approximate number of mosquito bites reported was not associated with mosquito protection measures taken $(\mathrm{p}>0.40)$. Of the 20 respondents that used no mosquito protection at all, the median number of mosquito bites was 17.5 (range: 0-100), while the median number of mosquito bites among the 19 respondents that protected themselves sometimes or always was 10 (range: $0-250$ ). Three participants who reported receiving "uncountable" or an unknown number of mosquito bites were not included in this analysis.

All of the nine participants that reported illness also reported receiving mosquito bites (range 5-100), but only four reported using protective measures (two DEET only, two clothing only). The cleaning methods employed in their rehabilitation facilities varied as well, with two disinfecting daily, four disinfecting only between cases, and two disinfecting only periodically. One participant with illness was unsure of the daily cleaning product used. The number of raptors admitted into the participants' facilities ranged from 5 to 290 (median 50) and included 2 to 98 (median 21) WNV-confirmed/suspected raptors. The use of gloves when handling raptors varied greatly as well. Leather gloves were worn by eight respondents, and latex gloves were worn by only four respondents. Only five of the respondents reported handwashing. Four reported wearing a face mask "when needed." Univariate and multivariate analyses found no association between developing illness and any of these potential risk factors $(\mathrm{p}>0.10)$.

\section{Discussion}

Based on the data collected in this survey, it is not possible to determine if any of the reported illnesses that occurred among rehabilitators during the 2002 WNV season were due to WNV infection. Nine participants reported illness during the summer of 2002. Only one ill participant sought medical care, and no diagnosis was made. Even if the rehabilitator illnesses had been due to WNV infection, it is unlikely the study would have been able to determine whether it was caused by exposure to sick birds. The number of mosquito bites received despite protecting against mosquito exposure was variable, and there is a lack of temporal sequence regarding this aspect of the study; it is unknown whether mosquito protective behaviors were performed before or as a result of receiving mosquito bites. This may have been a factor in our finding that taking mosquito protective measures did not seem to affect the number of mosquito bites received. Further studies evaluating the risk of WNV infection in personnel caring for wildlife are needed, particularly as potential non-mosquito modes of WNV transmission have since been reported (Komar 2003).

None of the risk factors evaluated in this study were found to be statistically significant for developing illness. The small sample size of the survey may have affected the findings of our analysis. In addition, there were a number of other limitations to this study, primarily selection and recall biases. Although the NWRA is a large organization to which many wildlife rehabilitators belong, there are an unknown number of rehabilitators who are not members and therefore may not have been adequately represented in the sample. Attempts to reach the wildlife rehabilitators selected were limited to those with a telephone number listed in the NWRA directory and to those available at that number during normal business hours (Monday through Friday, 9am to $5 \mathrm{pm}$ CST) or at least an answering machine or voicemail service. In addition, there may have been increased participation by rehabilitators who were aware of the reports of rehabilitator illness due to West Nile virus. Because the survey occurred up to two months after the WNV season had begun to wane in 2002, the rehabilitators' abilities to recall requested information may have led to misclassification.

Despite the limitations and statistical findings, this study does suggest that many wildlife rehabilitators may not be taking adequate precautions to minimize personal injury and illness nor the spread of disease among their admitted cases. Most veterinarians have received training in recognizing diseases of animals and their potential for transmission to humans and other animals; therefore, veterinarians who provide services to wildlife rehabilitation facilities are in a unique position to provide guidance regarding the protective measures that are needed when working with sick or injured wildlife, the importance of protecting their personal health, and the importance of seeking medical attention when symptoms of illness arise. The NWRA and International Wildlife Rehabilitation Council (IWRC) have included programs during their annual meetings and have published information on zoonoses and personal protection (Wolf 1995; Friend 2001; Miller 2000). However, there are rehabilitators who are not members of these organizations or any other rehabilitator organization. Without information from such external sources, these rehabilitators' prima- 
ry source of information would likely be their attending veterinarian and other local rehabilitators. Thus, it is important to provide training to rehabilitators not only at annual wildlife rehabilitation meetings, but also local meetings and their rehabilitation facilities.

For many veterinarians and rehabilitators, providing services to rehabilitation facilities is a second job that provides little or no pay. Therefore, there are constraints on their ability to spend time on issues other than treating the animals. Training in zoonoses transmission and prevention would, however, be time well spent with regard to animal, rehabilitator and veterinarian health and safety. The importance of preventing personal injury and the need for familiarity with potential disease agents carried by wild animals under care are of key importance for those regularly working and handling these animals (Hodge 1991, McLean 1994).

Variations exist between states regarding the requirements of wildlife rehabilitation permittees. Some state requirements may include an examination or interview requirement to evaluate knowledge of basic rehabilitation concepts, continuing education, and membership in NWRA or IWRC. Some states are very specific in their regulations for husbandry and infection control, while others are not. It may prove worthwhile in the future to investigate whether state regulations affect rehabilitator knowledge of infection control and worker health risks.

\section{LITERATURE CITED}

Friend, M. 2001. Wildlife Rehabilitation and Disease Emergence: Opportunities, Challenges and Responsibilities. Pp. 3-20 in Wildlife Rehabilitation, Vol. 19 (D. Ludwig, ed). National Wildlife Rehabilitators Association: St. Cloud, MN.

Hodge, G. 1991. The Pocket Guide to the Humane Control of Wildlife in Cities and Towns. The Humane Society of the United States.

Komar, N. 2003. West Nile Virus: Ecology and Epidemiology in North America. Advances in Virus Research 61:185-234.

McLean, R. G. 1994. Wildlife Diseases and Humans, in Prevention and Control of Wildlife Damage. Pp. A25-A41. Cooperative Extension, Institute of Agriculture and Natural Resources, University of Nebraska, Lincoln.

Miller, E. A., editor. 2000. Minimum Standards for Wildlife Rehabilitation, 3rd edition. National Wildlife Rehabilitators Association: St. Cloud, MN.

Wolf, L. 1995. Zoonoses: What you don't know can hurt you. NWRA Quarterly. 13(2):5-10. (沺)

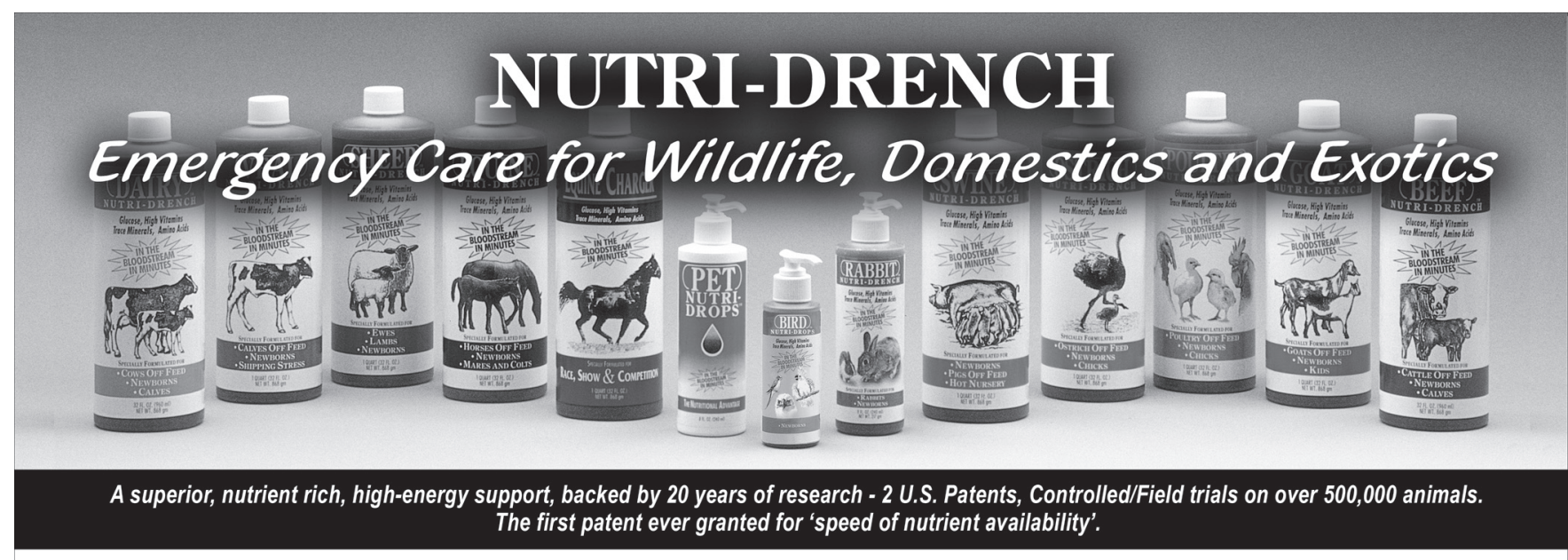

BoviDr Laboratories revolutionizes the industry with the first and only oral nutrition support to immediately restore the immune system. The speed is comparable to the injection of vital nutrients into the bloodstream with a hypodermic needle. Measurable in minutes; $50 \%$ uptake in 30 minutes.

- Increase survival for wildlife, domestics and exotics

- Energize weak incoming wildlife with uncertain health status

- Stimulate appetite during disease, after surgery or injury

- Correct diarrhea - $99 \%$ utilization of nutrients
- Calm behavior after trauma, high stress or transport

- Preventative for stress induced infection

- Restore the immune system with vital nutrients in perfect balance

- Maximize antibiotic response (elevates blood glucose levels)

Dramatic response in any stress condition when energy/replacement nutrition is needed fast ........ALL IN MINUTES! 